\title{
Kidney Segmentation in Three-Dimensional Ultrasound Images using a Fast Phase-based Approach
}

\author{
Helena R. Torres ${ }^{1,2,3,4}$, Sandro Queirós ${ }^{3,4^{*}}$, Pedro Morais ${ }^{3,4 *}$, Bruno Oliveira ${ }^{1,2,3,4}$, João Gomes- \\ Fonseca $^{3,4}$, Paulo Mota ${ }^{3,4}$, Estevão Lima ${ }^{3,4}$, Jan D’hooge ${ }^{5}$, Jaime C. Fonseca' ${ }^{2}$, João L. Vilaça ${ }^{1}$ \\ ${ }^{12} \mathrm{Ai}$ - Polytechnic Institute of Cávado and Ave, Barcelos, Portugal \\ ${ }^{2}$ Algoritmi Center, School of Engineering, University of Minho, Guimarães, Portugal \\ ${ }^{3}$ Life and Health Sciences Research Institute (ICVS), School of Medicine, University of Minho, Braga, Portugal \\ ${ }^{4}$ ICVS/3B's - PT Government Associate Laboratory, Braga/Guimarães, Portugal \\ ${ }^{5}$ Lab on Cardiovascular Imaging and Dynamics, KU Leuven, Belgium
}

\begin{abstract}
Renal ultrasound imaging is the primary imaging modality for the assessment of the kidney's condition and is essential for diagnosis, treatment and surgical intervention planning, and follow-up. In this regard, kidney delineation in three-dimensional ultrasound images represents a relevant and challenging task in clinical practice. In this paper, a novel framework is proposed to accurately segment the kidney in 3D ultrasound images. The proposed framework can be divided into two stages: 1) initialization of the segmentation method; and 2) kidney segmentation. Within the initialization stage, a phase-based feature detection method is used to detect edge points at kidney boundaries, from which the segmentation is automatically initialized. In the segmentation stage, the B-Spline Explicit Active Surface framework is adapted to obtain the final contour of the kidney. Here, a novel hybrid energy functional that combines localized region-based and edge-based terms is used during segmentation. For the edge term, a fast-modified phase-based detection approach is applied. The proposed framework was tested in 15 3D ultrasound images. The method showed to be accurate and feasible in very challenging poor-quality ultrasound images, achieving a Dice overlap of approximately $81 \%$ and an average point-to-surface error of $2.4 \mathrm{~mm}$. Moreover, a validation set composed of 24 additional images of healthy kidneys and 8 additional images of pathologic ones was evaluated to corroborate the accuracy of the method. The obtained results demonstrated the potential of the proposed method to be used in clinical practice.
\end{abstract}

Index Terms-B-spline Explicit Actives Surfaces, feature detection, kidney segmentation, 3D ultrasound

\section{INTRODUCTION}

Renal imaging systems play an important role in the analysis of the kidney's condition and are essential for diagnosis, follow-up, and planning/execution of renal interventions. Ultrasound (US) imaging is one of the most common imaging modalities to assess kidney function, being non-invasive, radiation-free, and providing a low-cost and real-time examination [1]. Plus, 3D US technology allows the acquisition of 3D volumetric data, enabling a more detailed analysis of the kidney and better understanding of its spatial information. However, US images usually have low image quality and exhibit speckle noise, low contrast, and artifacts, such as shadows and dropouts. Therefore, the task of segmenting the kidney in these images is challenging. In clinical practice, this task is often performed manually, and it is time-consuming, labor-intensive, and prone to inter and intra-observer variability. In this sense, an automatic or semi-automatic segmentation method can be helpful to design a computer-aided diagnostic tool to perform functional kidney evaluation, allowing to minimize manual interaction and reduce inter- and intra-observer variability. Such tool can be applied in different strands of clinical practice: 1) in diagnosis for extraction of information about the kidney and its condition; 2) in surgical planning for renal interventions; and 3) in the intervention itself, for guidance.

Kidney segmentation in three-dimensional US images is the driving problem discussed in this paper. Currently, semiautomatic and automatic methods have already been proposed in the literature for this purpose, including image-based approaches [2], [3], active shape/appearance models [4]-[7], atlas-based techniques [8], and deformable models [9]-[12]. An exhaustive review of kidney segmentation methods was performed by the proponent authors and it is presented in [13]. In short, Mendoza et al. presented a framework to segment the kidney using shape and appearance models [4], [5]. In [6], Cerrolaza et al. proposed a new variant of active appearance models, using Gabor features to create a Gabor-based appearance model. To increase the efficiency of the previous method, a Gabor-based fuzzy appearance model was proposed in [7] to deal with the intensity variability of US images. However, a manual definition of the kidney axis was still required to initialize the strategy. Different types of deformable models approaches were also proposed to segment the kidney. An extremely time-consuming fast marching method followed by an edge-based level-set method was initially used by Noll et al. [9]. Although interesting results were achieved, no anatomical information was included in the model, limiting its performance. In [10], [12], Marsousi et al. proposed a shapebased kidney segmentation method that uses a template matching technique to detect the kidney and then a region-based level-set segmentation method was applied. To improve the 
accuracy of the method, the same author added texture information extracted from Gabor filtering classifiers [8]. In [11], a deformable models framework was also used where a kidney shape model was globally and locally deformed using appearance information.

Despite the recurrent use of active contour methods in kidney segmentation, the poor quality of the ultrasound images remains a drawback for these traditional deformable models approaches, especially for the ones that only explore intensity information for the segmentation process. To tackle this issue, more recently, some authors proposed to use phase-based analysis within the deformable models approach in different medical areas [14]-[16], to deal with the characteristics of the US images. The main advantage of phase-based approaches is the lower sensitivity to noise, contrast distortions, and image illumination, as it is intensity invariant and therefore showing high accuracy and good response to speckle noise in US images. Nevertheless, these phase-based solutions were not explored for 3D US kidney segmentation. Plus, even though different solutions were already described, a fast, robust, and accurate solution to segment the kidney in 3D US data was not described, limiting its correct evaluation in traditional clinical practice.

The focus of the present work was to develop a kidney segmentation framework for 3D US images using a deformable model combined with a phase-based method. The main goals of the segmentation method were a low computational time and its robustness and accuracy in delineating the kidney. These aspects are important for the method to be applied for the abovementioned strands of clinical practice. Hereto, the main contributions in the present paper can be described as follows:

- A method to identify the kidney boundaries in 3D US images was developed. This method uses phase-based image information to create a robust edge map of the US image;

- A new image-based initialization method was developed to create an initial segmentation of the kidney, using the edge map information. This initial segmentation is used as an initial contour for the final segmentation method;

- The B-spline Explicit Active Surfaces (BEAS) framework originally proposed by Barbosa et al. [17] was extended for abdominal ultrasound images for the first time. This framework allows an accurate and real-time segmentation of heterogeneous images;

- A novel energy functional was developed. This energy functional combines a region-based term with an edge-based term to improve the accuracy of the BEAS method.

This paper is organized as follows. In section II, the proposed framework is described. In section III, the implementation details of the method are presented. In section IV, the experiments performed are described, being the results presented in section V. In section VI, the segmentation performance is discussed and in section VII the main conclusions of this paper are given.

\section{Methodology}

\section{A. General overview}

The first stage of the proposed method (Fig. 1) corresponds to a contour initialization method (section II.B) by identifying the kidney boundaries. From a manually given centroid of the kidney, optimal kidney boundaries are detected through a phase-detector approach and used to obtain the initial contour for the 3D segmentation model. The presence of outliers in these boundary positions is prevented through a Random Sample Consensus (RANSAC) method.

The second stage concerns the kidney segmentation method (section II.C). This block is based on the BEAS framework. To obtain an accurate 3D segmentation of the kidney, a hybrid energy functional that combines localized region-based and edge-based terms was developed. The edge-based term of the energy functional uses phase information of the image to improve the accuracy of the segmentation. This energy was designed to best fit the characteristics of the US images.

\section{B. Contour initialization using image-based features}

1) Phase-based feature detection

Image features, such as edges, corners, and ridges, are extremely important for several methods in image processing. To quantify the significance of these features, one needs to study the local properties of the signal, using its analytical signal. This analytical signal is created using the Hilbert Transform for a 1D case [18]. The Hilbert Transform extends a signal into the complex plane, allowing the calculation of the signal's amplitude and phase. However, the generalization of the Hilbert Transform to higher dimensions is not linear. To solve this problem, Felsberg and Sommer proposed an extension of the analytical signal, denominated monogenic signal [19]. Instead of being computed using the Hilbert Transform, the monogenic signal is computed using the Riesz Transform, which is a vector-valued extension of the Hilbert Transform. For feature measurement, this monogenic signal is usually represented by its scalar-valued even response and by its vector-valued odd response. The even and odd responses are given, respectively, by:

$$
\begin{gathered}
\operatorname{even}(x, y, z)=f(x, y, z) * g(x, y, z) ; \\
\operatorname{odd}(x, y, z)= \\
\sqrt{\sum_{d}\left(\left(f(x, y, z) * g(x, y, z) * h_{d}(x, y, z)\right)^{2}\right.},
\end{gathered}
$$

with $d=1,2,3, h_{d}(x, y, z)$ are the Riesz filters for each image $(f(x, y, z))$ dimension, as described in [19], and $g(x, y, z)$ is a band-pass zero-DC linear filter used to avoid undesirable frequencies and enhance spatial localization of the features. From these responses, it is possible to search for edge steps of the image by searching points of asymmetry in the image's spectrum. This asymmetry can be measured using the equation:

$$
F A(x, y, z)=\frac{\lfloor|\operatorname{odd}(x, y, z)|-|\operatorname{even}(x, y, z)|-T\rfloor}{\sqrt{\operatorname{odd}(x, y, z)^{2}+\operatorname{even}(x, y, z)^{2}+\varepsilon}},
$$


Initialization stage

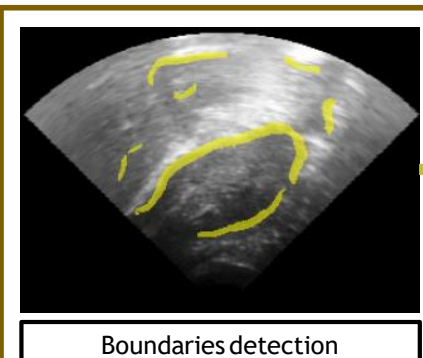

Boundaries detection

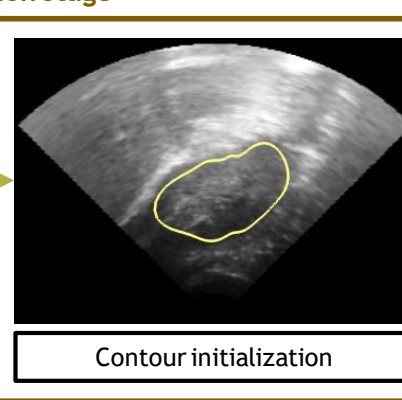

Segmentation stage

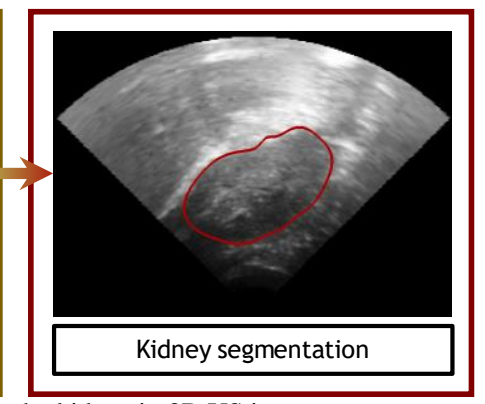

Fig. 1 - Overview of the proposed framework to segment the kidney in 3D US images.

where $\varepsilon$ is a small constant to avoid division by zero, the operator $[$.$\rfloor denotes zeroing negative values, and T$ is a threshold value for noise compensation estimated as described in [18].

\section{2) Kidney's boundary detection}

Using the feature asymmetry measure $F A$, it is possible to create an edge map of an image. This phase-based feature detection can be used to detect the boundaries of the kidney in the US image (Fig. 2). However, the conventional formulation of $F A$ has the drawback of outputting the same response for bright-to-dark and dark-to-bright edges (Fig. 2F). In this sense, different edges are detected inside the kidney, namely the transitions between the parenchyma and image background and between the renal pelvis and renal parenchyma. To solve this problem, a new formulation of the phase-based feature detection method is proposed. The first step of the proposed reformulation is to convert a filtered version of the US image in a polar representation, converting its Cartesian coordinates to a spherical coordinate system (Fig. 2C). In this system, each point of the image is determined by three parameters: the distance to a fixed reference point $(\rho)$, the angle in the zenithal direction $(\theta)$, and the angle in the azimuthal plane $(\varphi)$. A manually defined point within the renal pelvis (i.e. the kidney centroid) was set as the origin of the spherical coordinate system. In the spherical domain, the transitions between the renal parenchyma and the background are seen as dark-to-bright transitions as seen from the center of this coordinate system. To detect only these dark-to-bright transitions, a reformulated $F A$ measure was applied to the polar image:

$$
F A_{r}(\rho, \theta, \varphi)=\frac{\lfloor\mid \text { odd }(\rho, \theta, \varphi) \mid-\operatorname{even}(\rho, \theta, \varphi)-T\rfloor}{\sqrt{\operatorname{odd}(\rho, \theta, \varphi)^{2}+\operatorname{even}(\rho, \theta, \varphi)^{2}+\varepsilon}}
$$

This reformulation considered the characteristics of the odd and even responses. Indeed, in edge transitions, the magnitude of the odd response is large, while the magnitude of the even response is small. Moreover, the even response is computed by convolving the image with the chosen band-pass filter, resulting in higher responses in the brighter side of the edge transition and lower in the darker. In this sense, if one considers the real even response, instead of its absolute value, the $F A_{r}$ measure results in lower/higher responses in the brighter/darker regions near the transitions. Note that the odd and even responses were computed by convolving the monogenic signal of the spherical image with a band-pass zero-DC Difference-of-Gaussians (DoG) filter.
The next step to construct the edge map of the kidney's boundaries is to compute the finite differences of $F A_{r}$ in the radial direction:

$$
F A_{r_{\rho}}(\rho, \theta, \varphi)=F A_{r}(\rho, \theta, \varphi)-F A_{r}(\rho-1, \theta, \varphi),
$$

where the dark-to-bright transitions present negative values. The finite difference response is then used to restrict the $F A$ detector, where transitions with negative finite differences are maintained whereas the remaining ones are removed (Fig. 2E).

$$
F A_{D B}(\rho, \theta, \varphi)=\left\{\begin{array}{ll}
F A(\rho, \theta, \varphi), & \text { if } F A_{r_{\rho}}(\rho, \theta, \varphi)<0 \\
0, & \text { if } F A_{r_{\rho}}(\rho, \theta, \varphi) \geq 0
\end{array}\right. \text {. }
$$

The final step is to transform the resulting edge map to the original Cartesian system (Fig. 2G). This map is thresholded to obtain a binary image, allowing to remove edges with low phase responses that do not correspond to the kidney's boundaries.

\section{3) Contour initialization}

The created edge map contains the boundaries of the kidney and is used to initialize the contour for the segmentation process (Fig. 3). For that, a set of points belonging to the kidney boundaries are found through a ray tracing procedure, inspired by the work of Noll et al. [9]. In this procedure, rays are emitted from the kidney's center in angles that vary between 0 to 360 degrees in the azimuthal plane and -90 to 90 degrees in the zenithal plane, covering the entire region around the kidney (Fig. 3B). During this procedure, the first non-zero value encountered are used as kidney points. The main idea is to fit a surface to this set of points (Fig. 3C) and use the surface as initial contour, enabling to initialize the segmentation process with a contour very close to the true kidney's boundaries (Fig. $3 \mathrm{D})$. The main drawback in this initialization approach is the presence of outliers in the edge map that do not correspond to the kidney boundaries, which can lead to a sub-optimal representation of the kidney's shape. To solve this problem, the RANSAC [20] method was used to remove outliers points, using an ellipsoid as initial model. In short, an ellipsoid model is firstly fitted to the transition points found, which allows to identify a set of initial inlier points. Then, at each iteration of the RANSAC method, the ellipsoid model is refitted to the transition points using the Iterative Closest Point (ICP) algorithm [21], allowing to refine the search for the inlier points. The combination of RANSAC and ICP methods allows removing the outliers in the points detected by the rays, improving the initial contour for the segmentation process. 

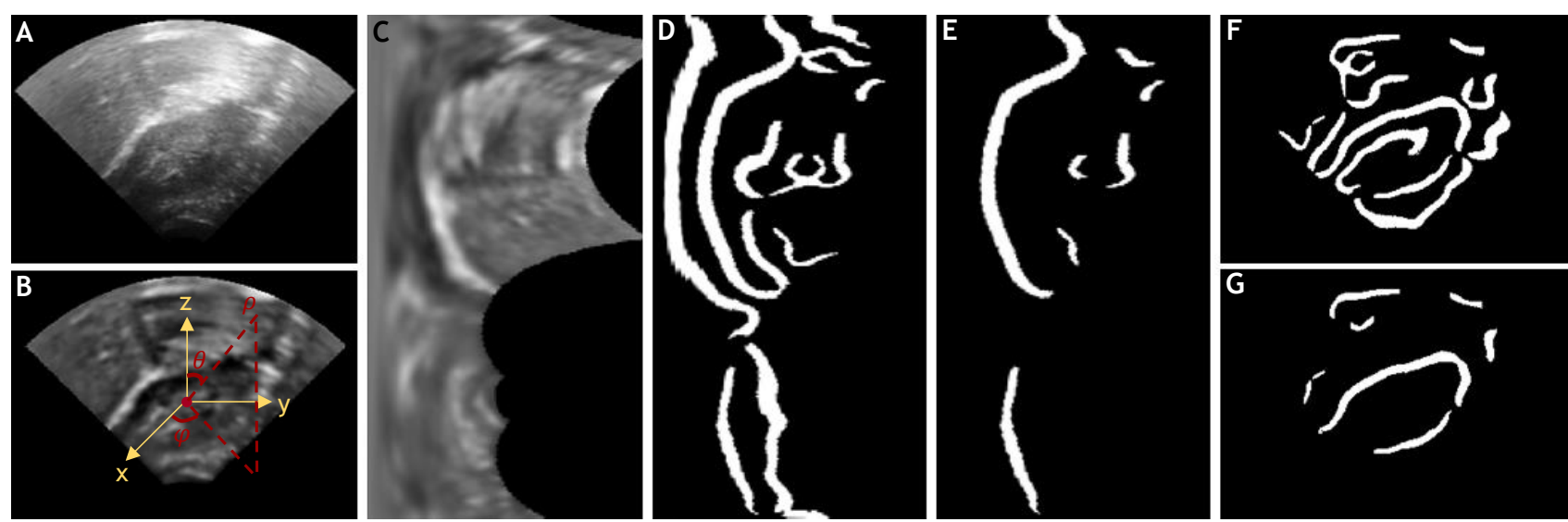

Fig. 2 - Edge maps computed using phase-based detection method. (A) Slice of the original US image; (B) Processed image with representation of the coordinate systems; (C) Polar representation of (B); (D) Detected edges using phase-based detection method; (E) Detected edges using the proposed modified phase-based detection method; (F) Cartesian representation of (D); (G) Cartesian representation of (E).
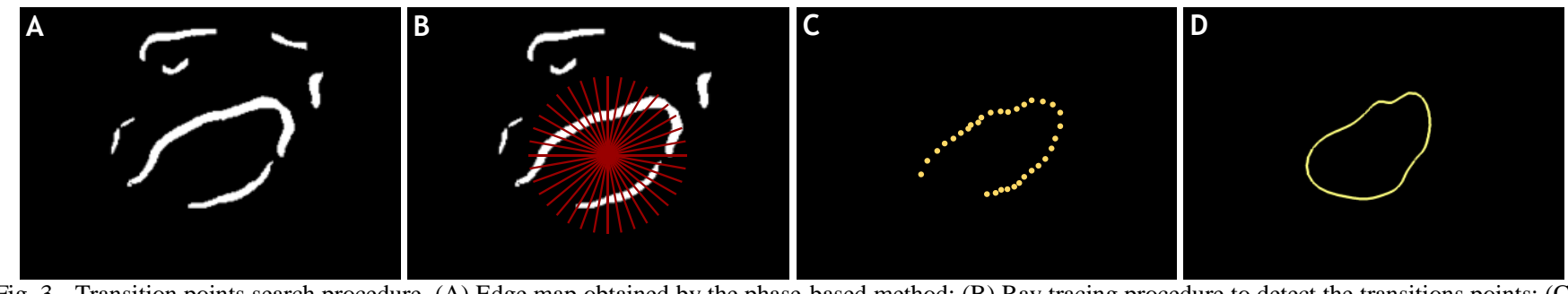

Fig. 3 - Transition points search procedure. (A) Edge map obtained by the phase-based method; (B) Ray tracing procedure to detect the transitions points; (C) Detected transition points; (D) Contour fitted to the transition points.

\section{Segmentation using B-spline Explicit Active Surfaces} $(B E A S)$

\section{1) B-spline Explicit Active Surfaces (BEAS)}

After contour initialization, the BEAS framework proposed by Barbosa et al. [17] was used to segment the kidney. The primary principle of the BEAS framework is the active contour method. In active contours, an evolving interface is propagated by minimizing a given energy functional that reflects the characteristics of the object to be segmented. In BEAS, the concept of Active Geometric Functions (AGF) [22] is used by representing the evolving interface as an explicit function. Geometrically, this implies that one of the coordinates of the points on a surface is given explicitly as a function of the remaining coordinates. Such explicit relation can be mathematically defined as:

$$
\psi: \mathbb{R}^{n-1} \mapsto \mathbb{R}, x_{1}=\psi\left(\boldsymbol{x}^{*}\right),
$$

where $\boldsymbol{x}$ is a point of coordinates $\left\{x_{1}, \ldots, x_{n}\right\}$ within the surface and $\boldsymbol{x}^{*}=\left\{x_{2}, \ldots, x_{n}\right\}$. This explicit form of the interface reduces the dimensionality of the segmentation problem, limiting the topology of the contour. Nevertheless, this formulation enables to recover the kidney boundary, owing to its convex and smooth shape.

The BEAS method extends the concept of AGF to the variational level-set B-spline formulation proposed in [23]. In this formulation, the interface function is modeled as a continuous parametric function expressed on a B-spline basis, where the minimization of the energy functional is expressed as a sequence of 1D convolutions. Therefore, the BEAS method expresses $\psi$ as a linear combination of B-spline basis functions:

$$
x_{1}=\psi\left(x_{2}, \ldots, x_{n}\right)=\sum_{k \in \mathbb{Z}^{n-1}} c[\boldsymbol{k}] \beta^{d}\left(\frac{\boldsymbol{x}^{*}}{h}-\boldsymbol{k}\right),
$$

where $\beta^{d}($.$) is the uniform symmetric (n-1)$-dimensional $\mathrm{B}$-spline of degree $d$. The knots of the $\mathrm{B}$-splines are located on a rectangular grid defined on the chosen coordinate system, with a regular spacing given by $h$. The coefficients of the Bspline representation are gathered in $c[\boldsymbol{k}]$ and the minimization of the energy functional can be directly obtained in terms of these coefficients.

\section{2) Energy functional}

To evolve the surface an appropriate energy criterion must be defined. In [24], Lankton and Tannenbaum introduced the concept of localized region-based energies. Using these energies, the evolving interface is propagated taking into account the properties of the local regions around each point of the interface. A localized region-based energy functional can be defined in terms of a generic force function $F$ as:

$$
E=\int_{\Omega} \delta_{\phi}(\boldsymbol{x}) \int_{\Omega} B(\boldsymbol{x}, \boldsymbol{y}) \cdot F(\boldsymbol{y}) d \boldsymbol{y} d \boldsymbol{x},
$$

where

$$
F(\boldsymbol{y})=f_{\text {in }}(\boldsymbol{y}) \cdot H_{\phi}(\boldsymbol{y})+f_{\text {out }}(\boldsymbol{y}) \cdot\left(1-H_{\phi}(\boldsymbol{y})\right),
$$

and $f_{\text {in }}$ and $f_{\text {out }}$ provide energy criteria for the interior and the exterior region of the interface $\Gamma . \delta_{\phi}(\boldsymbol{x})$ specifies the interface and $H_{\phi}(\boldsymbol{x})$ is the Heaviside function applied to the level-set like function $\phi(\boldsymbol{x})=\Gamma\left(\boldsymbol{x}^{*}\right)-x_{1}$, specifying the interior of the contour. The exterior of the contour is defined as $\left(1-H_{\phi}(\boldsymbol{x})\right)$. $B(\boldsymbol{x}, \boldsymbol{y})$ is a mask function in which the local parameters are 
estimated to drive the contour evolution. This mask is defined around each point $\boldsymbol{x}$ as the set of points belonging to the normal direction of $\boldsymbol{x}$ and whose distance is lower than a parameter $r$ :

$$
B(\boldsymbol{x}, \boldsymbol{y})=\left\{\begin{array}{ll}
1, & \text { if } \boldsymbol{y}=\boldsymbol{x}+k \times \widehat{\boldsymbol{N}}, k \in[-r, r] \\
0, & \text { otherwise }
\end{array} .\right.
$$

To minimize the abovementioned energy, a modified version of the gradient descent optimization with feedback step adjustment algorithm is used. In BEAS, the energy can be directly minimized with respect to $c[\boldsymbol{k}]$ through the following evolution equations:

$$
\begin{gathered}
c[\boldsymbol{k}]^{t+1}=c[\boldsymbol{k}]^{t}+\lambda \frac{\partial E}{\partial c[\boldsymbol{k}]^{(t)}}, \\
\frac{\partial E}{\partial c\left[\boldsymbol{k}_{\boldsymbol{i}}\right]}=\int_{\Gamma}\left(\bar{g}\left(\boldsymbol{x}^{*}\right)\right) \beta^{d}\left(\frac{\boldsymbol{x}^{*}}{h}-\boldsymbol{k}_{\boldsymbol{i}}\right) d x^{*},
\end{gathered}
$$

where $t$ is the current iteration number and $\lambda$ is a parameter controlling the step at each iteration. $\bar{g}\left(\boldsymbol{x}^{*}\right)$ is a feature function that reflects the features of the object to be segmented. For clarity's sake, considering a generic function $h(x)$ in $\mathbb{R}^{n,}, \bar{h}$ is noted as the restriction of $h$ over the interface $\Gamma$ in $\mathbb{R}^{n-1}$.

\section{3) Hybrid localized energy functional}

There are several region-based energy functionals proposed in the literature. In [25], Queirós et al. proposed a signed version of the Yezzi energy described in [26]. This energy evolves the contour to have the maximum separation between the image statistics, modeling the foreground and background regions as having maximally separated intensities. To explicitly specify the expected relation between both regions, the signed version of this energy is expressed in terms of $F$ as:

$$
F_{L S Y}(y)=u_{x}-v_{x}
$$

where $u_{x}$ and $v_{x}$ are the mean intensities inside and outside of the evolving contour at point $\boldsymbol{x}$. With this energy, the contour is forced to evolve so that the interior of the interface has lower intensities than its exterior, assuming that the object to be segmented is darker than the background. The feature function for the evolution is given by:

$$
\bar{g}_{L S Y}\left(\boldsymbol{x}^{*}\right)=\left(\bar{I}\left(\boldsymbol{x}^{*}\right)-u_{x}\right)+\left(\bar{I}\left(\boldsymbol{x}^{*}\right)-v_{x}\right)
$$

where $\bar{I}\left(\boldsymbol{x}^{*}\right)$ corresponds to the image value at point $\boldsymbol{x}$.

Since the kidney appears as a hypoechogenic structure in the US image and its surroundings have brighter intensities, the Signed Localized Yezzi (LSY) energy seems to be an adequate choice for its segmentation. However, the region-based energy alone may not be able to deal with the characteristics of the US image. Therefore, a localized edge-based energy (LP) that analysis the image using a phase-based approach, was created to improve the accuracy of the segmentation method. For that, we propose to use the $1 \mathrm{D}$ version of the $F A_{D B}$ measure to analyze the transitions of the signal given by the set of points belonging to the mask $B$. This $1 \mathrm{D}$ signal includes information about image features along the normal direction of each contour point, containing information about the intensity transitions that correspond to the kidney boundaries. The $1 \mathrm{D} F A_{D B}$ measure of the mask signals was computed using a $1 \mathrm{D}$ DoG filter. The feature response acts as a high-resolution phase-based edge indicator and was used as the localized edge-based energy itself, being expressed as:

$$
F_{L P}(y)=F A_{D B}\left(x^{*}\right) .
$$

This term presents its maximum in dark-to-bright intensity transitions and presents values equal to zero in the other regions of the signal. The feature function associated with this energy is computed by convolving the energy with the derivative of a Gaussian function $G$ :

$$
\bar{g}_{L P}\left(\boldsymbol{x}^{*}\right)=\nabla G * F A_{D B}\left(\boldsymbol{x}^{*}\right)
$$

The proposed energy functional consists in the combination of the region-based energy and the proposed edge-based energy (Fig. 4), resulting in a hybrid energy functional expressed as:

$$
F(\boldsymbol{y})=\alpha_{S} \cdot\left(u_{x}-v_{x}\right)-\beta_{S} \cdot F A_{D B}\left(\boldsymbol{x}^{*}\right),
$$

where $\alpha_{S}$ and $\beta_{S}$ are weight factors that control the influence of each term in contour evolution. The feature function associated with the proposed energy functional is given by:

$$
\begin{array}{r}
\bar{g}\left(\boldsymbol{x}^{*}\right)=\alpha_{S} \cdot\left(\left(\bar{I}\left(\boldsymbol{x}^{*}\right)-u_{x}\right)+\left(\bar{I}\left(\boldsymbol{x}^{*}\right)-v_{x}\right)\right) \\
+\beta_{S \cdot}\left(\nabla G * F A_{D B}\left(\boldsymbol{x}^{*}\right)\right)
\end{array}
$$

\section{IMPLEMENTATION DETAILS}

In the implementation of the proposed method, some parameters had to be defined. Concerning the contour initialization method, the spherical representation of the US image was obtained by setting the number of angles used in the both zenithal and azimuthal plane to 250 (a resolution of $\sim 1^{\circ}$ in the three-dimensional space), allowing to convert the Cartesian image without losing much detail. To obtain the binarized edge map, a threshold of 0.025 was applied to the original map, allowing to remove edges with very low phase responses. For the transition points search procedure, the number of rays was experimentally set to 128 for both zenithal and azimuthal planes. In the RANSAC method, the number of iterations was set to 10 and the data points were tested with respect to the chosen model in terms of distance, defining the points more than $5 \mathrm{~mm}$ away outliers. Note that these parameters were chosen after experimental evaluation.

Regarding the BEAS framework, an appropriate coordinate system was defined. Due to the kidney's ellipsoid shape, a spherical coordinate system was chosen. Thus, the radial coordinate of the points within the surface was given as a function of the azimuth and zenith angles. Concerning the Bspline scales and number of surface points, the first one was set to $2^{2}$ and 32 points were used to define each direction of the rectangular grid in the spherical domain. These values were chosen to obtain the shape detail needed, as shown in APPENDIX A. Regarding mask $B$, the radius of the normal vector was set to $10 \mathrm{~mm}$ to assess local statistics (please see APPENDIX A for a deeper insight regarding this parameter). Finally, the weights associated to the region and edge-based terms in the hybrid energy functional were set to 1 and 1.75 respectively. 

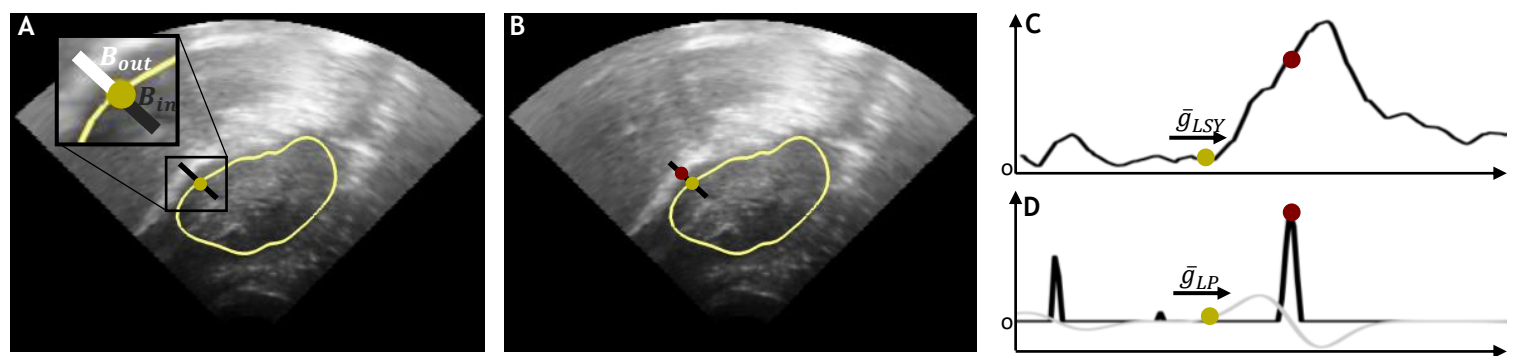

Fig. 4 - Principle of the localized energy functional used in the segmentation method. (A) Mask function separated in its interior region $\left(B_{\text {in }}\right)$ and exterior region $\left(B_{\text {out }}\right)$ from a surface point (yellow dot); (B) Evolving contour showing the position of a surface point (yellow dot) and the kidney transition (red dot); (C) Image intensity profile extracted in the mask function; (D) Result of the modified phase-based feature detection method applied in the signal represented in (C) used as energy functional (black line) and the respective feature function (grey line).

\section{EXPERIMENTS}

\section{A. Datasets description}

The performance of the proposed segmentation method was evaluated on 47 3D US images. The images were divided into three sets: one testing set composed of 15 healthy cases, one validation set with 24 additional images of healthy kidneys (validation set 1), and one validation set composed of 8 additional pathologic cases (validation set 2), including $X$ cases of renal lithiasis and $\mathrm{X}$ cases of renal masses. During algorithm testing, the testing set was used for different experiments (see section IV-C) and for the definition of the implementation parameters described in section III. The images in this testing set were acquired with the GE Vivid E9 ultrasound scanner with a patient-specific gain, depth, and time-gain compensation setup. The probe used is a matrix of array transducers and works in a range of frequencies between 1.5 and $4.0 \mathrm{MHz}$, which is adequate for deep structures such as the kidney. The remaining sets were used as validation sets for final method evaluation (see section IV-D). The images in both validation sets were acquired with the $\mathrm{X}$ ultrasound scanner and an abdominal probe with a frequency range of $\mathrm{X}-\mathrm{X} \mathrm{MHz}$ was used. The image size across all sets ranged from $265 \times 267 \times 185$ to $318 \times 300 \times 203$, with an isotropic voxel spacing ranging from 0.49 to $0.92 \mathrm{~mm}$.

\section{B. Ground truth}

The segmentation results were compared against manual delineations performed by an experienced urologist. The manual delineation was performed in the MITK software [27]. Multiple 2D slices were manually selected and segmented by the expert and then interpolated to obtain a final 3D surface. After 3D interpolation, 2D corrections were performed if required. To study the inter-observer variability and compare it with the segmentation results, a second observer manually segmented the kidney in the testing set. Thus, it was possible to compare the accuracy of the segmentation method with the reproducibility between observers.

\section{Method Evaluation}

To initialize the proposed method, a user selected one point in the interior of the renal pelvis to generate the initial contour for the segmentation process. In the segmentation method, the proposed hybrid energy functional (LSY + LP) was compared with the LSY energy.
To study the robustness of the proposed method against the initial point, different initial points were evaluated. For that, 100 points were tested per image. These points were generated by randomly translating the point defined by the user by a maximum of $1 \mathrm{~cm}$ in any direction. Moreover, the proposed method was also evaluated against a manual initialization of the contour. Here, the user selected fourteen points in the kidney and an ellipsoid was fitted to this data, being used as an initial contour for the segmentation process. Please note that the abovementioned experiments were conducted in the testing set.

Concerning the evaluation metrics, the Dice coefficient, average point-to-surface distance (P2S), 95 ${ }^{\text {th }}$ Hausdorff distance (HD), and percentage of "good contours" (GC, which accounts for the percentage of cases for which the mean P2S is lower than $5 \mathrm{~mm}$ ) were used to assess method's performance.

\section{Validation experiment}

As a final experiment, the proposed segmentation method was applied to 24 additional images of healthy kidneys and to 8 additional images of pathological ones. In this final validation of the proposed method, the abovementioned implementation parameters were kept, allowing to assess the method's performance on new clinical data without new parameter search. Once the images used in the testing and validation sets differ from each other's with respect to image quality (different ultrasound scanners and probes were used), the feasibility of using the proposed approach to new clinical data is assessed, allowing to study method's robustness and generalization. The same evaluation metrics from the abovementioned experiments were used in this validation experiment.

\section{RESULTS}

TABLE I summarizes the performance of the method in the testing set assessed in terms of Dice, P2S, HD, and GC. The performance of the proposed energy functional was compared with the LSY energy, whose results are also in the table. Moreover, the inter-observer variability is presented. In Fig. 5, the boxplots for each evaluation metric are presented to analyze the performance distribution in terms of percentiles and outlier points. In Fig. 6, three different examples of the segmentation results are presented: a good segmentation result, an intermediate, and a bad one.

As stated, the method's robustness with respect to the initial points was tested and the results are presented in TABLE II. In the table, the results for different initial points are presented and 
TABLE I.

3D KIDNEY SEGMENTATION PERFORMANCE IN THE TESTING SET $(\mu \pm \sigma)$

\begin{tabular}{ccccc}
\hline \hline Energy & Dice (\%) & P2S (mm) & HD (mm) & GC (\%) \\
\hline Initialization & $71.8 \pm 6.5$ & $3.6 \pm 1.1$ & $9.2 \pm 3.0$ & 93.3 \\
LSY & $79.4 \pm 7.0$ & $2.6 \pm 1.1$ & $8.5 \pm 3.6$ & 93.3 \\
LSY+LP & $81.0 \pm 5.8$ & $2.4 \pm 0.9$ & $8.0 \pm 3.2$ & 100.0 \\
\hline Inter-observer & $84.6 \pm 5.0$ & $2.7 \pm 0.8$ & $7.7 \pm 3.3$ & 100.0 \\
\hline \hline
\end{tabular}

compared with the results obtained with the user-defined point. However, $6.6 \%$ of the points randomly generated could not be used once the initial contour presented $\mathrm{P} 2 \mathrm{~S}$ errors higher than $7.5 \mathrm{~mm}$, which can be explained by its wrong location in relation to the renal pelvis. In this sense, these points were eliminated and the results for these points are not accounted in the table. In TABLE III, the proposed method is compared with a manual initialization of the contour given by an ellipsoid.

In TABLE IV, the method performance in the validation sets is presented, while one segmentation result example for each is shown in Fig. 7. Moreover, a comparison with state-of-the-art methods is presented in TABLE $\mathrm{V}$.

Regarding the method's computational burden, the average computational time is reported in TABLE VI. The computational time was assessed separately for each stage of the method. This framework was implemented in MATLAB and a 3.6 GHz Core computer with CPU i7-4740 and 16 GB of RAM.

\section{DISCUSSION}

This paper proposes a segmentation approach to extract the kidney from 3D US images, which makes use of a phase-based feature detection method and a deformable model framework. Our findings showed the accuracy of both initialization and segmentation stages. The contour initialization method achieved a Dice overlap of $\sim 72 \%$ (TABLE I), proving its accuracy. For the segmentation stage, TABLE I shows the accuracy of the method with a Dice overlap of $81 \%$ and a P2S distance lower than $2.5 \mathrm{~mm}$, which are good segmentation results given the inter-observer variability and the overall poorquality US image. All segmentations were considered "good contours". The proposed hybrid energy functional showed to outperform the LSY energy. When using the latter, the segmentation evolution maximizes the difference between the intensities inside and outside of the contour, forcing lower intensities inside than outside. Although this energy allowed decreasing the probability of finding local minima in comparison with other energies described in the literature, and although it resulted in acceptable segmentations (Dice of $\sim 79 \%$ ), it could overall not deal sufficient well with the characteristics of an ultrasound image. The addition of the proposed edge-based term allowed decreasing the errors of the segmentation results. This is explained by the phase-based edge term itself, which forces the surface to evolve towards positions with higher values of the feature asymmetry measure, i.e. the kidney boundaries. This term is crucial to quickly push the contour when far from the true boundaries, therefore increasing the capture range of the proposed method. When closer to the boundary, the result is mainly refined through the localized region-based energy. Moreover, since the edge-based term searches for specific transitions (dark-to-bright), the presence of surrounding interfaces is penalized, making the method more robust against poor initialization. One possible limitation emerges in cases of kidney's boundaries occlusion due to image dropouts or shadowing artifacts. In cases where only partial edges are occluded, the smoothing properties of the B-spline formulation allows to compensate missing information. However, in cases of high absence of image information at kidney's boundaries, the full shape of the kidney may not be recovered. This can be solved by adding a shape-based term to the BEAS framework, where shape knowledge, retrieved e.g. from a statistical shape model, can be used to constraint the deformable model-based approach, allowing to obtain a more reliable delineation. In fact, this solution is expected to be explored in the future. Nevertheless, the obtained results showed the accuracy of the segmentation method in the tested images. From TABLE I, the segmentation results can also be compared with the inter-variability between observers. The inter-observer agreement in terms of Dice metric was 84.6\%. Thus, the accuracy of the proposed method was only $3.6 \%$ less than the inter-observer variability and therefore it can be concluded that the performance of the method is reasonably close to the variability between different manual segmentations.

Regarding the initialization method, TABLE II allows to study the robustness of this stage to different initial points. Firstly, note that $6.6 \%$ of the total random points used to initialize the contours resulted in bad initial contours. This can be explained due to the specifications of the initialization method. Once the method searches for dark-to-bright transitions, the initial point must be given in the brighter region of the kidney, i.e. the renal pelvis. If the initial point is given too far from this region, the transitions between the pelvis and the parenchyma are also seen as dark-to-bright transitions, instead of bright-to-dark. Thus, the initial contour may be stuck at these internal boundaries of the kidney. Nevertheless, this fraction of bad initial contours can be considered small as only $\sim 7$ points per image failed to provide good initial contours. To not be biased by these bad initial contours, these points were not included in TABLE II. The results obtained for different initial points were similar to the ones obtained with the initial point given by the user, allowing to conclude the feasibility of the proposed method.

Besides this study to evaluate the robustness of the initialization method to the initial point, a different study was conducted to compare the segmentation performance against an initial contour manually given. The proposed method outperformed a manual initialization process (Table III). This can be explained by the fact that the initial contour generated by the proposed method was very close to the expected final contour. Thus, this initial contour was more representative of the kidney's true shape than a simplification of the common kidney's shape (i.e. ellipsoidal shape).

When validating the proposed method in new clinical cases, the segmentation performance showed in TABLE IV corroborates the robustness of the method and its generalization to different image types, in both healthy and pathological kidneys. Interestingly, a higher DICE was obtained for the validation set composed of healthy kidneys. Concerning the performance on the pathological validation set, the method's 

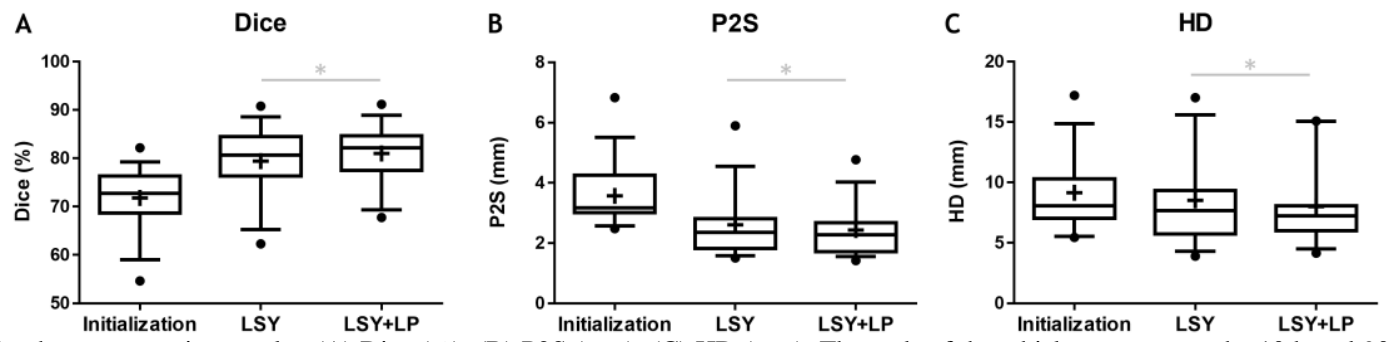

Fig. 5 - Boxplots for the segmentation results. (A) Dice (\%); (B) P2S (mm); (C) HD (mm). The ends of the whiskers represent the 10th and 90th percentiles. The crosses represent the mean values for each metric. The data points considered as outlier are plotted as dots. ${ }^{*} p<0.05$ in a two-tailed paired $t$-test assessed between LSY and LSY+LP.
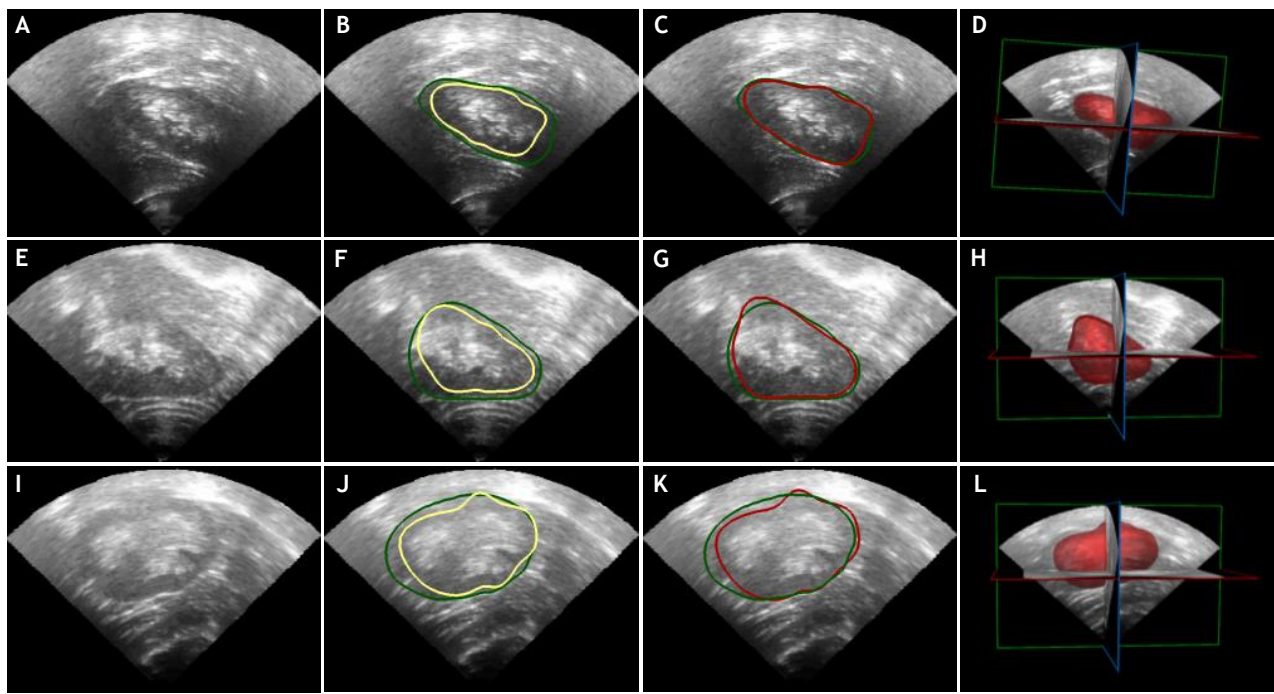

Fig. 6 - Segmentation results in the testing dataset using the proposed energy functional. (A), (E) and (I) Slice of the original images; (B), (F) and (J) Initial contour; (C), (G) and (K) Segmentation result; (D), (H) and (L) Surface of the segmentation result. The ground truth, the initial contour, and the segmentation results are delineated in green, yellow, and red, respectively.

TABLE II.

3D KIDNEY SEGMENTATION PERFORMANCE IN THE TESTING SET FOR DIFFERENT INITIAL POINTS $(\mu \pm \sigma)$

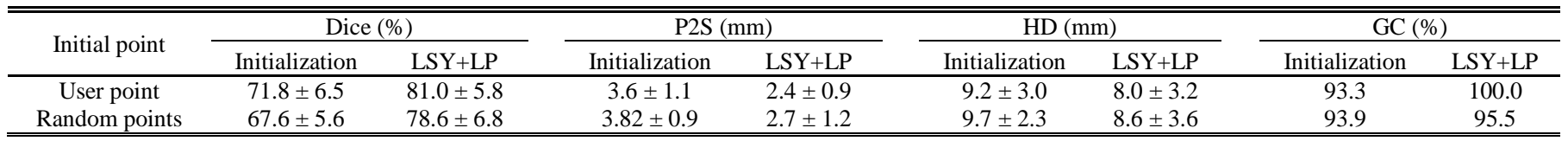

TABLE III.

3D KIDNEY SEGMENTATION PERFORMANCE IN THE TESTING SET FOR DIFFERENT INITIAL CONTOURS $(\mu \pm \sigma)$

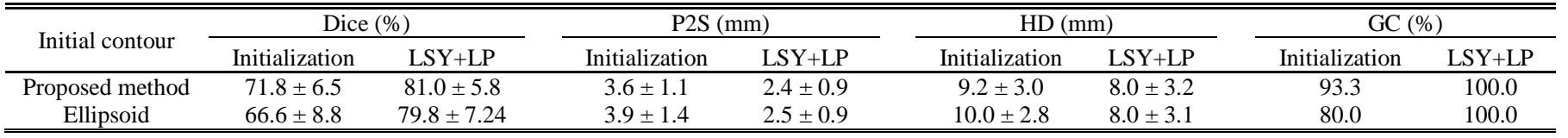

TABLE IV.

3D KIDNEY SEGMENTATION PERFORMANCE IN THE VALIDATION SETS $(\mu \pm \sigma)$

\begin{tabular}{ccccc}
\hline \hline Dataset & DICE $(\%)$ & P2S (mm) & HD $(\mathrm{mm})$ & GC (\%) \\
\hline Validation set 1 & $82.6 \pm 3.4$ & $2.8 \pm 0.7$ & $8.5 \pm 2.5$ & 100.0 \\
Validation set 2 & $78.8 \pm 6.5$ & $3.1 \pm 1.0$ & $9.4 \pm 3.6$ & 100.0 \\
\hline \hline
\end{tabular}

accuracy in terms of DICE was slightly worst. However, once the evaluated pathological images present foreign structures inside the kidney (such as stones and tumors) that can hamper the segmentation process, the decrease of the method's performance is expected. Nevertheless, the results obtained showed the method's accuracy even for these images, where all segmentations were considered "good contours".

The performance of the proposed method in all sets also showed to be competitive with the state-of-the-art (see TABLE v). Comparing with the work of Noll et al. [9], and according to [12], the proposed framework outperforms that method by a significant $39 \%$ of Dice score. A similar conclusion is retrieved when compared with the work of Marsousi et al. in [10], where the Dice coefficient was $15 \%$ lower. In the work of Marsousi et al. [12], the reported segmentation performance was similar to the accuracy of the proposed method. Comparing with the work of Cerrolaza et al. [6], [7], the author reported a Dice coefficient of $85 \%$ and $86 \%$, respectively, which is higher than the segmentation accuracy of the proposed method. However, these approaches require a training dataset, making the accuracy of the algorithm dependent on the training data. Moreover, it should be noticed that these different methods were tested on independent clinical databases (TABLE IV), making a true, direct comparison impossible. The generation of a general benchmark could be an interesting contribution in this field, allowing correct comparison between different methods and potentiating the application of these techniques in the clinical field. 

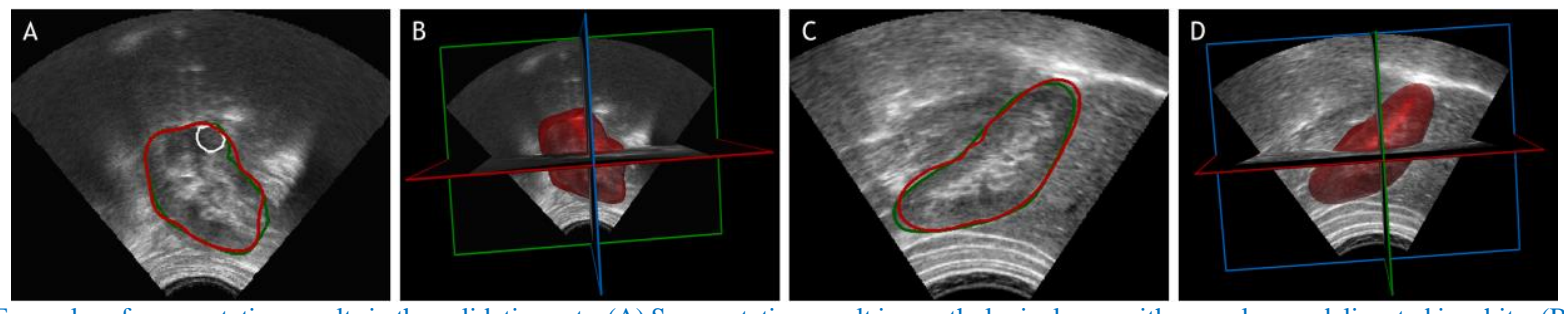

Fig. 7 - Examples of segmentation results in the validation sets. (A) Segmentation result in a pathological case with a renal mass delineated in white; (B) Surface of the segmentation result in the pathological case; (C) Segmentation result in a healthy case; (D) Surface of the segmentation result in the pathological case;

TABLE V.

COMPARISON WITH STATE-OF-THE-ART METHODS $(\mu \pm \sigma)$

\begin{tabular}{cccc}
\hline \hline Method & \# images & User interaction & Dice (\%) \\
\hline Proposed & 47 & $\mathrm{~S}$ & $81 \pm 5$ \\
Noll et al. [9] & 15 & $\mathrm{~A}$ & $42 \pm 8$ \\
Marsousi et al. [10] & 10 & $\mathrm{~A}$ & $66 \pm 6$ \\
Marsousi et al. [12] & 15 & $\mathrm{~A}$ & $81 \pm 4$ \\
Cerroloza et al. [6] & 14 & $\mathrm{~S}$ & $85 \pm 3$ \\
Cerroloza et al. [7] & 39 & $\mathrm{~S}$ & $86 \pm 3$ \\
\hline \hline
\end{tabular}

TABLE VI.

COMPUTATIONAL ANALYSIS OF THE PROPOSED METHOD $(\mu \pm \sigma)$

\begin{tabular}{ccc}
\hline \hline Stage & Time $(\mathrm{s})$ & BEAS iterations \\
\hline Initialization & $21.3 \pm 4.8$ & - \\
Segmentation & $3.6 \pm 1.6$ & $92.50 \pm 35.6$ \\
\hline \hline
\end{tabular}

The computational load of a method is important to understand its feasibility to be applied in clinical practice, namely for real-time interventions. The contour initialization method took on average $21 \mathrm{~s}$, being more than half of this time (13 s) related to the RANSAC method. The segmentation process was fast and took on average less than 3.6s, proving the clear advantages of the BEAS framework for fast 3D segmentation problems. Overall, the method took on average less than $25 \mathrm{~s}$ with a non-optimized code. This shows the feasibility for diagnostic use and surgical planning. Concerning real-time interventions, while the kidney must be segmented during successive frames, the initialization stage can be applied only once during the acquisition. Indeed, after contour initialization in the first frame, the segmentation obtained can be used as initial contour in the following frame. Hence, the computational cost of the contour initialization stage may not be a concern for a first approach of the method. Moreover, the implementation of the proposed framework can be optimized, and the computational time can be improved by applying a combination of multi-core multi-thread programming in $\mathrm{C}$. In this sense, the proposed pipeline for 3D kidney segmentation shows potential for its future application in renal interventions.

\section{CONCLUSION}

This paper presented a framework to segment the kidney in 3D US images. In summary, the proposed method can be divided into contour initialization and segmentation stages. The proposed approach was evaluated in 47 US images and showed to be accurate, robust, and competitive with the state-of-the-art. Regarding the initialization stage, the proposed method showed robustness to the initial point. Concerning the segmentation stage, the proposed hybrid energy functional showed high accuracy and feasible to deal with the characteristics of a US image. Moreover, the method proved to be computationally efficient, taking on average less than 25 s to segment the kidney. Overall, the proposed segmentation method proved to be successful and showed potential to be used in clinical practice.

In future work, it is intended to perform an extensive evaluation of the method in a higher database. Plus, we will develop an automatic kidney detection algorithm to fully automatize the segmentation and, thus, obtain a userindependent approach. This detection method must detect the renal pelvis in order to use the center of the renal structure as initial point for the proposed framework. Moreover, a specific segmentation module for the renal pelvis will be developed, thus increasing the interest of the proposed framework.

\section{APPENDIX A}

\section{STUDY OF BEAS PARAMETERS}

As stated, the BEAS parameters must be defined according to the segmentation problem. The choice of the number of surface points used is explicitly linked with B-spline scale, being paramount to define an ideal relation between these two parameters. The B-spline scales are related to the computation of the B-spline coefficients, which implicitly control the surface smoothing. By maintaining an adequate number of points, it is possible to vary the B-spline scale to obtain the ideal detail of the kidney's shape. On one hand, a low value may result in a jagged (i.e. non-smooth) contour, while increasing the method's computational load. On the other hand, if a high value is used, details of the real boundary of the kidney may be lost due to over-smoothing. Another BEAS parameter that must be defined is the size of the mask $B$, which must be defined according to the object to be segmented. On one hand, a small mask size may increase the probability of the segmentation to fall into local minima. On the other hand, a high value increases the area of the local regions around each point of the contour where the image statistics will be analyzed, making the method more sensitive to the artifacts of the US image. In Fig. 8, it is possible to observe the segmentation performance for different values of the B-spline scale and mask size. For this experiment, the number of surface points was set to 32, with the B-spline scale values ranging from $0^{2}$ to $4^{2}$ and the mask size varying from $5 \mathrm{~mm}$ to $30 \mathrm{~mm}$. In Fig. 9, the computational burden of the method for the experiment conducted is also presented.

Analyzing both Fig. 8 and Fig. 9, it is possible to verify that the ideal relation between the parameters is obtained by setting the B-spline scale to $2^{2}$ and the mask size to $10 \mathrm{~mm}$. In fact, good segmentation results were obtained even if the mask size varies from 10 to $16 \mathrm{~mm}$. However, an increase of the method's computational burden is verified when increasing the mask size. 


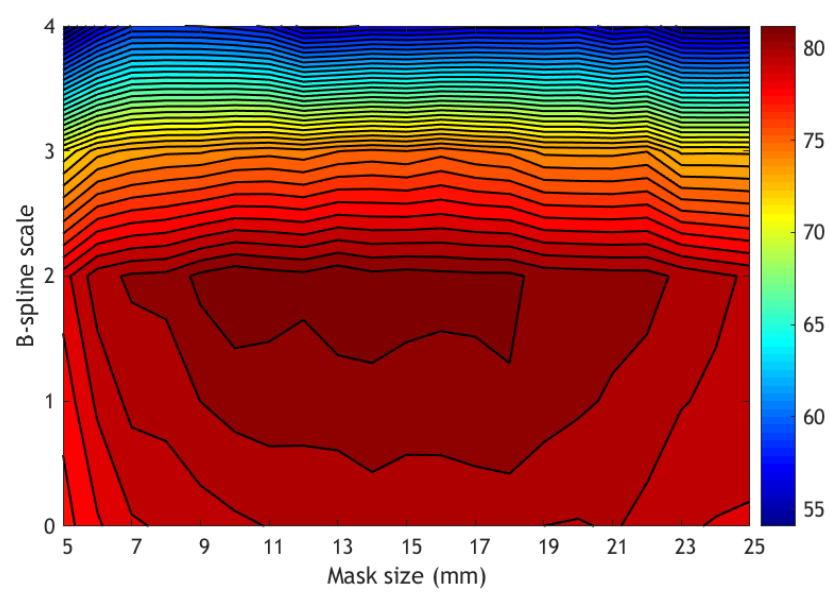

Fig. 8 - Performance of the segmentation method in the testing dataset in terms of Dice (\%) while varying B-spline scale and mask size.

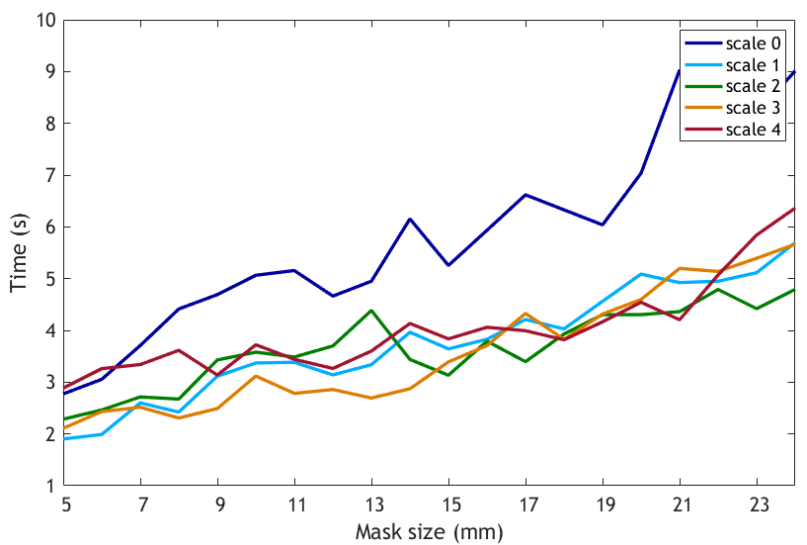

Fig. 9 - Computational burden of the segmentation stage varying B-spline scale and mask size.

Thus, with the defined parameters' values, a good compromise is achieved between segmentation performance and computational burden.

\section{REFERENCES}

[1] C. Vincent and P. Anahi, "Basics of Ultrasound Imaging," in Atlas of Ultrasound-Guided Procedures in Interventional Pain Management, S. N. Narouze, Ed. Springer New York, 2011, pp. 13-19.

[2] W. Mahani, H. Mahmud, R. Mohd, A. Raja, and E. Supriyanto, "Boundary Detection of Kidney Ultrasound Image Based on Vector Graphic Approach," J. Eng. Appl. Sci., vol. 10, no. 19, pp. 8822-8829, 2015.

[3] C. H. Wu and Y. N. Sun, "Segmentation of kidney from ultrasound B-mode images with texture-based classification," Comput. Methods Programs Biomed., vol. 84, no. 2-3, pp. 114-123, 2006.

[4] C. Mendoza et al., "Kidney segmentation in ultrasound via genetic initialization and active shape models with rotation correction," in International Symposium on Biomedical Imaging (ISBI), 2013, pp. 69-72.

[5] C. Mendoza et al., "Automatic analysis of pediatric renal ultrasound using shape, anatomical and image acquisition priors," in Medical Image Computing and Computer-Assisted Intervention (MICCAI), 2013, pp. 259266.

[6] J. J. Cerrolaza, N. Safdar, C. A. Peters, E. Myers, J. Jago, and M. G. Linguraru, "Segmentation of Kidney in 3D-Ultrasound Images Using Gabor-based Appearance Models," in International Symposium on Biomedical Imaging (ISBI), 2014, pp. 633-636.

[7] J. J. Cerrolaza, N. Safdar, E. Biggs, J. Jago, C. A. Peters, and M. G. Linguraru, "Renal Segmentation from 3D Ultrasound via Fuzzy Appearance Models and Patient-Specific Alpha Shapes," IEEE Trans. Med. Imaging, vol. 62, pp. 2393-2402, 2016.

[8] M. Marsousi, K. N. Plataniotis, and S. Stergiopoulos, "Atlas-Based Segmentation of Abdominal Organs in 3D Ultrasound, and its Application in Automated Kidney Segmentation," in IEEE Engineering in Medicine and Biology Society (EMBC), 2015, pp. 2001-2005.

[9] M. Noll, X. Li, and S. Wesarg, "Automated Kidney Detection and Segmentation in 3D Ultrasound," in Workshop on Clinical Image-Based Procedures, 2014, pp. 83-90.

[10] M. Marsousi, K. N. Plataniotis, and S. Stergiopoulos, "Shape-based kidney detection and segmentation in three-dimensional abdominal ultrasound images," IEEE Engineering in Medicine and Biology Society (EMBC). pp. 2890-2894, 2014.

[11]R. Ardon, R. Cuingnet, K. Bacchuwar, and V. Auvray, "Fast kidney detection and segmentation with learned kernel convolution and model deformation in 3D ultrasound images," in International Symposium on Biomedical Imaging (ISBI), 2015, pp. 268-271.

[12]M. Marsousi, K. Plataniotis, and S. Stergiopoulos, "An Automated Approach for Kidney Segmentation in Three-Dimensional Ultrasound Images," IEEE J. Biomed. Heal. Informatics, vol. 2194, pp. 1-15, 2016.

[13] H. R. Torres, S. Queirós, P. Morais, B. Oliveira, J. C. Fonseca, and J. L. Vilaça, "Kidney segmentation in ultrasound, magnetic resonance and computed tomography images: A systematic review," Comput. Methods Programs Biomed., vol. 157, pp. 49-67, Apr. 2018.

[14]A. Belaid, D. Boukerroui, Y. Maingourd, and J.-F. Lerallut, "Phase-based level set segmentation of ultrasound images.," IEEE Trans. Inf. Technol. Biomed., vol. 15, no. 1, pp. 138-147, 2011.

[15]W. Qiu et al., "Automatic segmentation approach to extracting neonatal cerebral ventricles from 3D ultrasound images," Med. Image Anal., vol. 35, pp. 181-191, 2017.

[16]L. Gao, X. Liu, and W. Chen, "Phase- and GVF-based level set segmentation of ultrasonic breast tumors," J. Appl. Math., 2012.

[17]D. Barbosa, T. Dietenbeck, J. Schaerer, J. D'hooge, D. Friboulet, and O. Bernard, "B-Spline Explicit Active Surfaces: An Efficient Framework for Real-Time 3-D Region-Based Segmentation," IEEE Trans. Image Process., vol. 21, no. 1, pp. 241-251, 2012.

[18]P. Kovesi, "Phase congruency: A low-level image invariant," Psychol. Res., vol. 64, no. 2, pp. 136-148, 2000.

[19]M. Felsberg and G. Sommer, "The monogenic signal," IEEE Trans. Signal Process., vol. 49, no. 12, pp. 3136-3144, 2001.

[20] M. a. Fischler and R. C. Bolles, "Random sample consensus: a paradigm for model fitting with applications to image analysis and automated cartography," Commun. ACM, vol. 24, no. 6, pp. 381-395, 1981.

[21]P. Besl and N. McKay, "A Method for Registration of 3-D Shapes," IEEE Transactions on Pattern Analysis and Machine Intelligence, vol. 14, no. 2. pp. 239-256, 1992.

[22]Q. Duan, E. D. Angelinib, and A. F. Lainea, "Real-time segmentation by Active Geometric Functions," Comput. Methods Programs Biomed., vol. 98, no. 3, pp. 223-230, 2011.

[23] O. Bernard, D. Friboulet, P. Thévenaz, and M. Unser, "Variational B-spline level-set: a linear filtering approach for fast deformable model evolution.," IEEE Trans. image Process., vol. 18, no. 6, pp. 1179-1191, 2009.

[24]S. Lankton and A. Tannenbaum, "Localizing Region-Based Active Contours," IEEE Trans. Image Process., vol. 17, no. 11, pp. 2029-2039, 2008.

[25]S. Queirós et al., "Fast automatic myocardial segmentation in 4D cine CMR datasets," Med. Image Anal., vol. 18, no. 7, pp. 1115-1131, 2014.

[26]A. Yezzi, A. Tsai, and A. Willsky, "A Fully Global Approach to Image Segmentation via Coupled Curve Evolution Equations," J. Vis. Commun. Image Represent., vol. 13, no. 1-2, pp. 195-216, 2002.

[27]I. Wolf et al., "The Medical Imaging Interaction Toolkit (MITK) - a toolkit facilitating the creation of interactive software by extending VTK and ITK," Med. Imaging 2004, pp. 16-27, 2004. 